\section{Efficient band-edge light injection in two- dimensional planar photonic crystals using a gradual interface}

\author{
Damien Bernier, Eric Cassan, Xavier Le Roux, \\ Delphine Marris-Morini, and Laurent Vivien \\ Université Paris-Sud-XI, Institut d'Electronique \\ Fondamentale, Bât 220 IEF Centre National de la \\ Recherche Scientifique UMR 8622, 91405 Orsay, France \\ E-mail: eric.cassan@ief.u-psud.fr
}

\begin{abstract}
Experimental results about efficient band-edge light coupling into two-dimensional planar photonic crystals by the use of a gradual interface are reported. It is shown that a chirp of both the lattice period and the hole diameter on short lengths below $3 \mu \mathrm{m}$ allows maintaining high optical transmission spectra close to the band-gap frequency where strongly dispersive phenomena can be exploited. If compared with nonoptimized structures, increased optical levels by 15 to $20 \mathrm{~dB}$ are obtained using gradual tapering stages. (๑) 2009 Society of Photo-Optical Instrumentation Engineers.
\end{abstract} [DOI: 10.1117/1.3184619]

Subject terms: photonic crystals (PhCs); dispersive properties; impedance mismatch; light coupling; gradual interface.

Paper 090275LR received Apr. 16, 2009; revised manuscript received Jun. 4, 2009; accepted for publication Jun. 8, 2009; published online Jul. 28, 2009

Photonic crystals (PhCs), which are periodically corrugated optical media, have received a growing interest in recent years due to their ability to tailor light properties at the wavelength scale. Most of these efforts have been devoted to photonic band-gap properties for light guiding and design of ultra-high-quality factor cavities. ${ }^{1-3}$ More recenty, attention has been paid to the strong dispersive properties of planar photonic crystals. ${ }^{4,5}$ Collimation, superlensing, and superprism phenomena have been reported in $\mathrm{PhCs}$ using specific tailoring of the $\mathrm{PhC}$ isofrequency surfaces. ${ }^{5,6}$ Such properties have opened opportunities to control the propagation direction and the profiles of optical beams in periodically corrugated planar optical media.

In most cases, light is injected from a slab optical waveguide and impinges at the interface between this homogeneous medium and the $\mathrm{PhC}$ area, leading to mechanisms of light refraction through the excitation of evancescent and propagative Bloch modes in the periodic medium. Beam shaping properties can be exploited only if a significant fraction of incident light power is coupled into the $\mathrm{PhC}$ area, i.e., if the impedance mismatch between the two media has been carefully taken into account using some tapering strategy. ${ }^{7}$

Previous works have adressed this problem in the case of light coupling from optical strip waveguides to $\mathrm{PhC}$ waveguides, ${ }^{8-10}$ but only a few works have specifically adressed this problem in the case of light coupling into

0091-3286/2009/\$25.00 @ 2009 SPIE half-space photonic crystals. In Ref. 11, an immitance matching strategy has been proposed to minimize the reflected power at the interface with semi-infinite PhCs. Momeni and co-workers have also proposed an adiabatic matching approach for coupling of light into planar $\mathrm{PhCs},{ }^{12}$ while Lee and co-workers have explored a method based on anti-reflection PhC layers. ${ }^{13}$ Modifying the shapes of holes was also proposed in Ref. 14, but the technological feasiblity of etching such holes was then not considered. Moreover, all these works have adressed the problem only from a theoretical point of view.

In this letter, we report experimental results showing the benefit of a gradual $\mathrm{PhC}$ interface to increase optical power transmission in periodically corrugated media up to the first PhC band-gap frequency of strong index contrast slab $\mathrm{PhCs}$, i.e., in the slow light regime. Among the possible applications, this could serve to strengthen optical nonlinear effects in integrated optical devices.

Experimental optical transmission across a nonoptimized square $\mathrm{PhC}$ area, i.e., with no gradual interface, is presented first. In a second step, it is compared with the same $\mathrm{PhC}$ region optimized with gradual stages at both input and output ends.

Figure 1 shows a scanning electron microscope (SEM) view of the realized experimental device. It consists of a 300-nm lattice parameter square $\mathrm{PhC}$ of circular airholes of normalized radius $r / a=0.26$ etched in a silicon-oninsulator (SOI) wafer with a 220 -nm-thick top silicon film separated from the $\mathrm{Si}$ substrate by a $1-\mu \mathrm{m} \mathrm{SiO}_{2}$ layer. $\mathrm{Re}-$ fractive slab waveguides were defined using a RAITH150 electron beam lithography process using negative resist. The $\mathrm{PhC}$ structure was separately insolated by means of a lithography process with positive resist. The photoresist patterns were transferred to the 150 -nm-thick top silica cladding layer using a reactive ion etching system. This layer served as a mask to etch the silicon film through a $\mathrm{SF}_{6} / \mathrm{O}_{2}$ anisotropic etching process.

The $\mathrm{PhC}$ area is made of 39 rows of holes, and the input and output SOI strip waveguides have widths of $12 \mu \mathrm{m}$ and $20 \mu \mathrm{m}$, respectively. These waveguides allow light injection and light collection, respectively, in the perpendicular direction with respect to the $\mathrm{PhC}$ lattice interface, i.e., in the $\Gamma-\mathrm{X}$ direction.

Figure 2 shows the square $\mathrm{PhC}$ dispersion diagram calculated using the 3-D guided-wave expansion method reported in Ref. 15 In the wavelength range of interest above $1500 \mathrm{~nm}$, the normalized frequency $a / \lambda$ is typically lower


Fig. 1 (a) Large-scale SEM view of the realized $\mathrm{PhC}$ inserted in a $100 \mu \mathrm{m} \times 100 \mu \mathrm{m}$ slab waveguide area with $12-\mu \mathrm{m}$-wide input and $20 \mu \mathrm{m}$-wide output SOI strip waveguides: the output waveguide width was chosen to ensure full light collection in spite of beam divergence occuring in the slab waveguide area after the $\mathrm{PhC}$ region. (b) Detailed SEM view of the PhC area. 


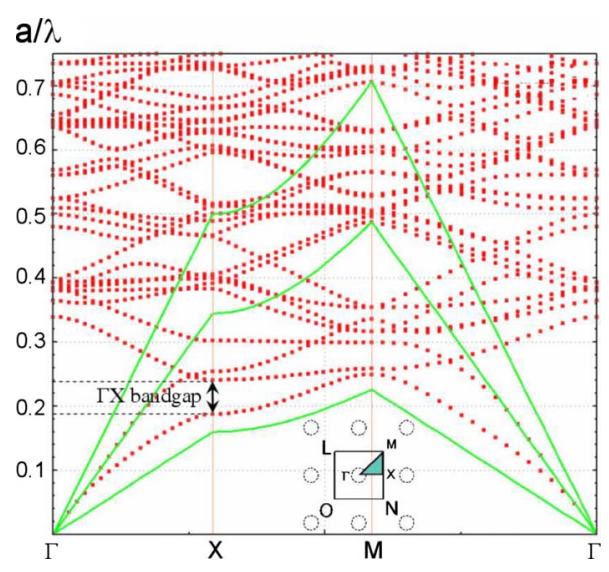

Fig. 2 Dispersion diagram of an asymmetrical planar airhole SOI PhC (bottom cladding is oxide and top cladding is air) calculated using the 3-D guided-wave expansion method described in Ref. 15. Silicon, oxide, and air lines are visible.

than 0.2 , and a photonic band gap is visible in the $\Gamma-\mathrm{X}$ direction near the first band of the square $\mathrm{PhC}$.

The experimental characterization was based on a setup using a tunable semiconductor laser in the spectral range between 1575 and $1625 \mathrm{~nm}$. A linearly polarized light beam was coupled into an input waveguide with a polarization-maintaining lensed-fiber using a butt-coupling technique. The output light was collected by a $40 \times$ objective and was measured with a large-area IR detector.

The exact value of the hole diameter was obtained from the comparison of the $\Gamma-\mathrm{X}$ band-gap frequency estimated using 3-D finite difference time domain (FDTD) simulations and from experimental optical transmission spectra. Figure 3 shows this comparison for the best hole diameter of $d=155 \mathrm{~nm}$. High-frequency fringes are visible in the experimental curve due to parasistic Fabry-Pérot reflections that have been hereafter filtered.

Light coupling at the edge of band 1 was further investigated by considering the possible tapering of the interface. As pointed out in Ref. 12, light reflection at the input/

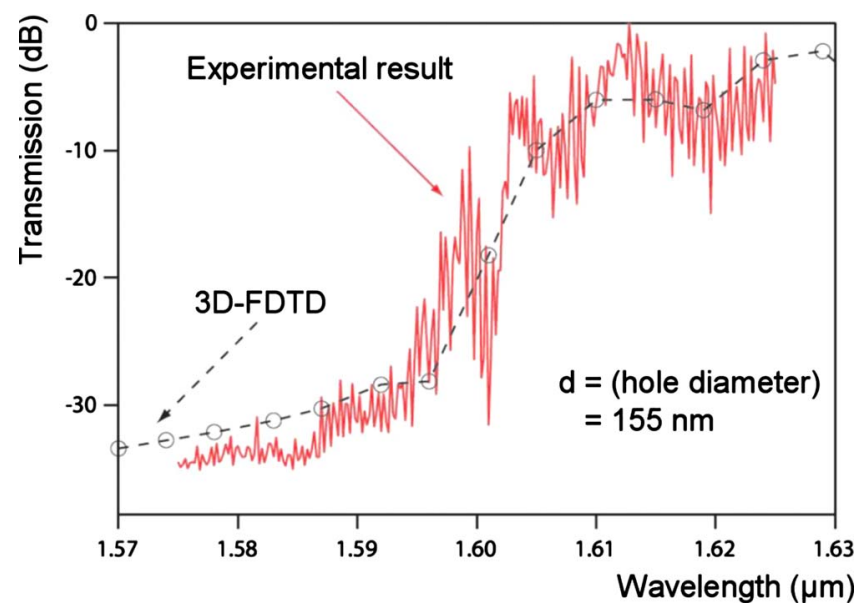

Fig. 3 Comparison between the 3-D FDTD calculated spectrum of the device described in Fig. 1 and the related experimental optical spectrum showing the good agreement between simulation and sample characterization for $d=155 \mathrm{~nm}$.

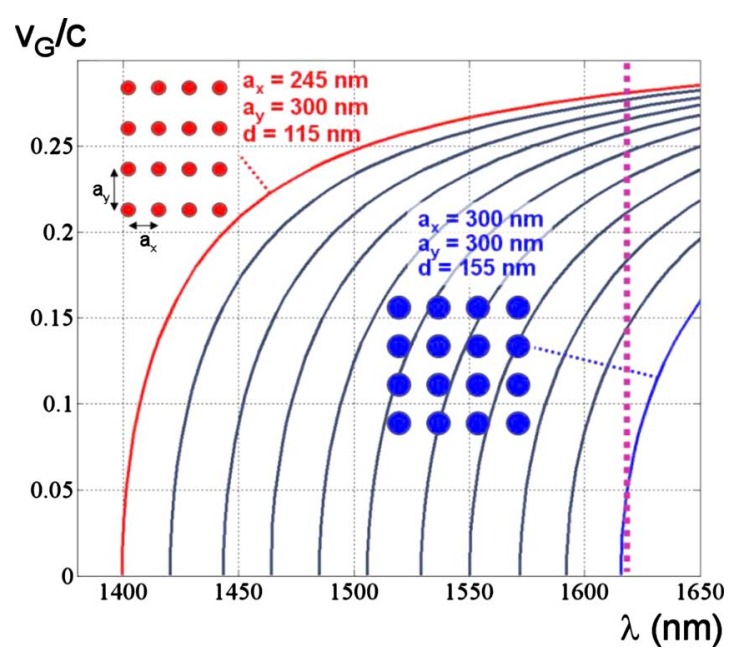

Fig. 4 Light group velocity in the $\Gamma-X$ direction for 10 different PhCs. The lattice parameter and hole diameter values of these PhCs have been chosen to correspond to those of the 10 rows of holes of the input and ouput tapers of the device shown in Fig. 5.

output interfaces near the PhC band edge is primarily due to group velocity mismatch between fast and slow waves. Here, the fast mode is related to the slab waveguide mode excited in the $100 \mu \mathrm{m} \times 100 \mu \mathrm{m}$ slab waveguide area before the light arrival at the interface with the $\mathrm{PhC}$ area.

In order to increase the light coupling efficiency within the $\mathrm{PhC}$ area, 10 rows of holes were added at both input and output interfaces. From the desired $\mathrm{PhC}$ lattice at the center, a chirp was introduced in the $\Gamma-\mathrm{X}$ direction (only) for both the lattice parameter and hole diameter, respectively. The lattice parameter in the $n$ 'th row of holes $(10 \geq n \geq 1))$ was taken as $98 \%$ of the previous value obtained for the $(n-1)$ 'th one. A similar chirp was introduced for the hole diameter, but with a $97 \%$ decrease rate instead of $98 \%$. The final lattice parameter and hole diameter values were then $245 \mathrm{~nm}$ and $115 \mathrm{~nm}$, respectively.

Figure 4 shows the calculated light group velocity for the first band in the $\Gamma-\mathrm{X}$ direction for the 10 different $\mathrm{PhCs}$ corresponding to the sets of lattice period and hole diameter values of the input/output tapering stages. It highlights the operation of gradual decrease of light group velocity in order to suppress the impedance mismatch between fast and slow waves at the interface between the slab waveguide and the 2-D PhC area.

For the practical realization of the $\mathrm{PhC}$ device, proximity effects during e-beam lithography steps were taken into account. We realized for this a specific characterization allowing the estimation of the correct resist exposure to minimize proximity effects. ${ }^{16}$ The whole technological process allowed the fabrication of graded $\mathrm{PhCs}$, i.e., fabrication tolerances did not prevent the possible use of physical effects due to lattice and hole diameter chirps. PhC.

Figure 5 shows an SEM view of the realized chirped

Figure 6 shows the experimental comparison between the initial $\mathrm{PhC}$ structure (without tapers) and the final $\mathrm{PhC}$ one (with tapers). It shows the benefit coming from the use of a gradual interface to efficiently inject light in the periodically corrugated optical medium. In this case, high- 


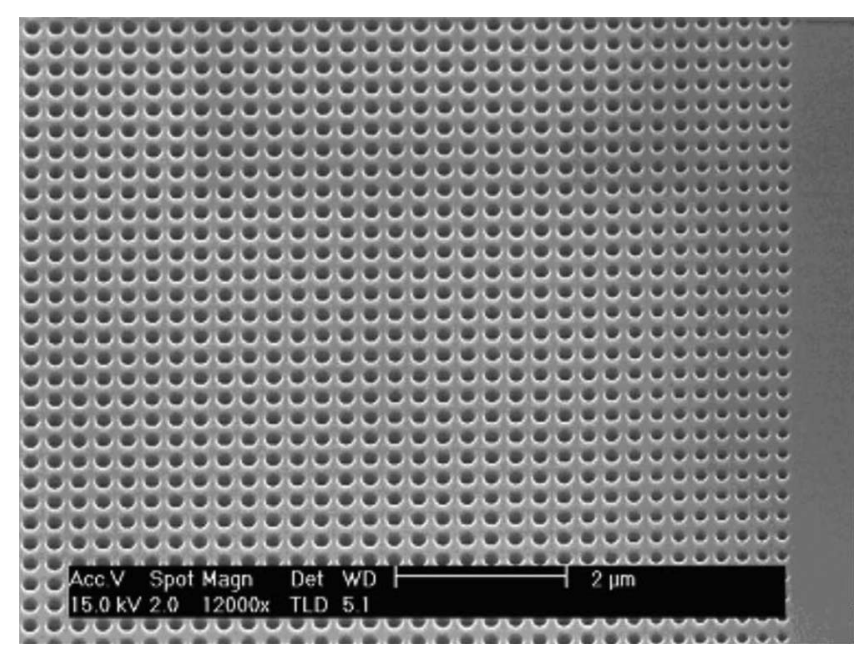

Fig. 5 SEM view of the chirped PhC output interface after correction of proximity effects: $98 \%$ and $97 \%$ of lattice and hole diameter chirps, respectively, have been considered from each additional row of holes for the last 10 rows of holes.

frequency $\left(>0.8 \mathrm{~nm}^{-1}\right)$ oscillations, due to parasistic Fabry-Pérot cavities were removed from the experimental results.

To conclude, planar $\mathrm{PhCs}$ have interesting properties to control light beam profiles and dispersion properties, but light has to be first efficiently injected into periodic media. We report in this paper experimental results about the possible use of a gradual interface to inject light in semi-

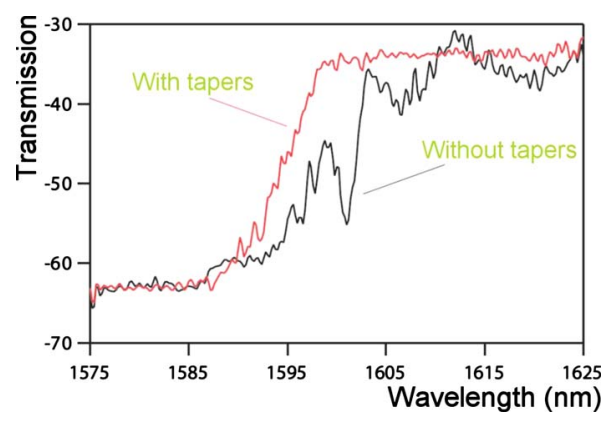

Fig. 6 Comparison between experimental optical transmission spectra with and without the optical tapers described in the captions of Fig. 4 and Fig. 5. infinite $\mathrm{PhC}$ areas. This approach is an efficient way to maximize light coupling into corrugated media, at least when power reflection is due to a group velocity mismatch between different regions. Such a result shows the possibility of exciting Bloch waves of planar PhCs close the photonic band-gap edge. This is interesting from a practical point of view, as most giant dispersive effects are obtained in this wavelength regime.

\section{References}

1. S. J. McNab, N. Moli, and Y. A. Vlasov, "Ultra-low loss photonic integrated circuit with membrane-type photonic crystal waveguides," Opt. Express 11(22), 2927-2939 (2003).

2. A. Y. Petrov and M. Eich, "Zero dispersion at small group velocities in photonic crystal waveguides," Appl. Phys. Lett. 85(21), 4866-4868 (2004).

3. Y. Akahane, T. Asano, and B.-S. Song, "High-Q photonic nanocavity in a two-dimensional photonic crystal," Nature (London) 425, 944947 (2003)

4. L. Wu, M. Mazilu, J.-F. Gallet, and T. F. Krauss, "Square lattice photonic crystal collimator," Photonics Nanostruct. Fundam. Appl. 1 31-36 (2003).

5. B. Momeni, J. Huang, M. Soltani, M. Askari, S. Mohammadi, M. Rakhshandehroo, and A. Adibi, "Compact wavelength demultiplexing using focusing negative index photonic crystal superprisms," Opt. Express 14(6), 2413-2422 (2006).

6. D. Bernier, X. Le Roux, A. Lupu, D. Marris-Morini, L. Vivien, and E. Cassan, "Compact, low cross-talk CWDM demultiplexer using photonic crystal superprism," Opt. Express 16(22), 17209-17214 (2008).

7. S. G. Johnson, P. Bienstman, M. A. Skorobogatiy, M. Ibanescu, E. Lidorikis, and J. D. Joannopoulos, "Adiabatic theorem and continuous coupled-mode theory for efficient taper transitions in photonic crystals," Phys. Rev. E 66, 066608 (2002).

8. A. Mekis and J. D. Joannopoulos, "Tapered couplers for efficient interfacing between dielectric and photonic crystal waveguides," $J$. Lightwave Technol. 19(6), 861-865 (2001).

9. T. Baba, D. Mori, K. Inoshita, and Y. Kuroki, "Light localizations in photonic crystal line defect waveguides," IEEE J. Sel. Top. Quantum Electron. 10(3), 484-491 (2004).

10. R. Biswas, Z. Y. Li, and K. M. Ho, "Impedance of photonic crystals and photonic crystal waveguides," Appl. Phys. Lett. 84(8), 12541256 (2004).

11. J. Ushida, M. Tokushima, M. Shirane, A. Gomyo, and H. Yamada, "Immitance matching for multidimensional open-system photonic crystals," Phys. Rev. B 68, 155115 (2003).

12. B. Momeni and A. Adibi, "Adiabatic matching stage for coupling of light to extended Bloch modes of photonic crystals," Appl. Phys. Lett. 87, 171104 (2005).

13. S.-G. Lee, J.-S. Choi, J.-E. Kim, and H. Y. Park, "Reflection minimization at two-dimensional photonic crystal interfaces," Opt. Express 16(6), 4270-4277 (2008).

14. T. Baba and D. Oshaki, "Interfaces of photonic crystals for high efficiency light transmission," Jpn. J. Appl. Phys., Part 1 40, 59205924 (2001).

15. Guided Mode Expansion (GME) software: http://fisicavolta.unipv.it/ dipartimento/ricerca/Fotonici/Index.htm.

16. R. Wüest, P. Strassera, M. Jungob, F. Robina, D. Ernib, and H. Jäckela, "An efficient proximity-effect correction method for electronbeam patterning of photonic-crystal devices," Microelectron. Eng. 67-68, 182-188 (2003). 